Recepción: 06 / 04 / 2018

Aceptación: 15 / 05 / 2018

Publicación: 02 / 07 / 2018

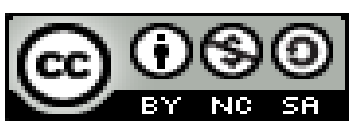

Ciencias técnicas y aplicadas

Artículo de Investigación

\title{
Análisis situacional del tratamiento de aceites automotrices residuales
}

\section{Situational analysis of the treatment of waste automotive oils}

\section{Análise situacional do tratamento de óleos automotivos residuais}

\author{
Celin A. Padilla-Padilla ${ }^{\mathrm{I}}$ \\ celin.padilla@espoch.edu.ec \\ Ligia E. Moreno-Pinduisaca ${ }^{\text {II }}$ \\ ligia.moreno@espoch.edu.ec \\ Luis F. Buenaño-Moyano III \\ lfbuenaño@espoch.edu.ec \\ Bolívar A. Cuaical-Angulo IV \\ bcuaical@espoch.edu.ec Olga \\ B. Barrera-Cárdenas ${ }^{\mathrm{V}}$ \\ olga.barrera@espoch.edu.ec
}

Correspondencia: celin.padilla@espoch.edu.ec

\begin{abstract}
${ }^{I}$ Magíster en Diseño Mecánico Mención en Fabricación de Autopartes de Vehículos, Master en Ingeniería de Vehículos Híbridos y Eléctricos, Diplomado Superior en Gestión del Aprendizaje Universitario, Ingeniero Automotriz, Facultad de Mecánica, Escuela Ingeniería Automotriz, Docente de la Escuela Superior Politécnica de Chimborazo, Riobamba, Ecuador.

${ }^{\text {II }}$ Magister en Gerencia y Liderazgo Educacional, Ingeniera Mecánica, Docente de la Escuela Superior Politécnica de Chimborazo, Riobamba, Ecuador.

III Magister en Gestión del Mantenimiento Industrial, Ingeniero Automotriz, Facultad de Mecánica, Escuela Ingeniería Automotriz, Docente de la Escuela Superior Politécnica de Chimborazo, Riobamba, Ecuador.

IV Magister en Gestión de Energías, Ingeniero Automotriz, Facultad de Mecánica, Escuela Ingeniería Automotriz, Docente de la Escuela Superior Politécnica de Chimborazo, Riobamba, Ecuador.

v Magíster En Matemática Básica, Magister En Docencia Universitaria E Investigación Educativa, Especialista En Computación Aplicada Al Ejercicio Docente, Doctora en Matemáticas, Facultad de Mecánica, Escuela Ingeniería Automotriz, Docente de la Escuela Superior Politécnica de Chimborazo, Riobamba, Ecuador.
\end{abstract}




\title{
Resumen
}

Este proyecto se enfoca en contribuir a la preservación del medio ambiente, la atención médica y la integridad personal de los actores involucrados en la generación de aceites lubricantes usados, que son una de las mayores fuentes de contaminación, tanto en aguas residuales, así como también en el suelo de las lubricadoras y talleres en la zona urbana del cantón Riobamba. El objetivo fue investigar los procedimientos para el manejo de los aceites utilizados en los talleres y lubricadoras automotrices. Se utilizó una metodología de tipo descriptiva de la contaminación ambiental producida por el manejo irresponsable de estos residuos. La población estuvo conformada por los talleres y lubricadoras de mayor afluencia del cantón Riobamba. Los resultados se obtuvieron a partir de visitas monitoreadas de seguimiento del destino de los aceites residuales en desuso por los automotrices. En la que se concluyó que los daños ambientales ocasionados por los residuos de aceites tanto en aguas residuales como en el suelo, se han dado por varios aspectos: falta de conocimiento de procedimientos técnicos para el manejo adecuado de este residuo y la composición química que estos poseen.

Palabras clave: aceite; contaminación; suelo; ambiente; taller; lubricadora.

\begin{abstract}
This project focuses on contributing to the preservation of the environment, medical care and personal integrity of the actors involved in the generation of used lubricating oils, which are one of the greatest sources of contamination, both in wastewater, as well as on the floor of lubricators and workshops in the urban area of the Riobamba canton. The objective was to investigate the procedures for the handling of oils used in automotive workshops and lubricators. A descriptive methodology of environmental pollution produced by the irresponsible management of this waste was used. The population was conformed by the workshops and lubricadoras of greater affluence of the Riobamba canton. The results were obtained from monitored visits to track the fate of waste oils in disuse by automotive. In which it was concluded that the environmental damage caused by waste oil in both wastewater and soil, have been given by several aspects: lack of knowledge of technical procedures for the proper handling of this waste and the chemical composition that these possess.
\end{abstract}

Keywords: Oil; contamination; floor; ambient; workshop; lubricator. 


\section{Resumo}

Este projeto tem como foco contribuir para a preservação do meio ambiente, cuidados médicos e integridade pessoal dos atores envolvidos na geração de óleos lubrificantes usados, que são uma das maiores fontes de contaminação, tanto em águas residuais, quanto no chão de lubrificadores e oficinas na área urbana do cantão de Riobamba. O objetivo foi investigar os procedimentos para o manuseio de óleos utilizados em oficinas automotivas e lubrificadores. Utilizou-se uma metodologia descritiva de poluição ambiental produzida pela gestão irresponsável desses resíduos. A população foi conformada pelas oficinas e lubrificadoras de maior afluencia do cantão de Riobamba. Os resultados foram obtidos a partir de visitas monitoradas para rastrear o destino dos óleos usados em desuso pelo setor automotivo. Em que se concluiu que os danos ambientais causados pelo óleo usado tanto nas águas residuárias como no solo, foram dados por vários aspectos: falta de conhecimento dos procedimentos técnicos para o manuseio correto desses resíduos e da composição química que estes possuir.

Palavras chave: Petróleo; poluição; solo; meio ambiente; oficina; lubrificador.

\section{Introducción}

En la industria automotriz se emplean grandes volúmenes de lubricantes, que se emplean en motores, caja de velocidades y diferenciales, además de otros elementos utilizados como grasas, líquido refrigerante, y otros líquidos empleados en el automóvil, estos líquidos son empleados para realizar diferentes funciones como la de lubricar, refrigerar o activación de algún mecanismo como lo hace el líquido de frenos. Todos los sistemas descritos anteriormente necesitan realizar un mantenimiento periódico, teniendo que se deseche los elementos que ya se han utilizado, específicamente lo referente a nuestro proyecto es el aceite, determinando el manejo que se realiza en este líquido, también revisar la reutilización adecuada. (Suntaxi Beltrán, 2012)

El índice de contaminación ambiental es uno de los problemas más importantes y comunes que se presentan en todo el mundo, los aceites residuales generados por los diferentes vehículos tanto livianos como pesados, así como también maquinas estacionarias utilizadas en termoeléctricas, petroleras, entre otras, representan más del 60\% de los aceites lubricantes consumidos, esto hace que los aceites usados sean uno de los residuos contaminantes más abundantes que se generan 
actualmente, pudiendo alcanzarse la cifra de 24 millones de toneladas al año. (Martínez Pérez, 2011).

En Ecuador, ha tenido un notable incremento en el parque automotor, en el último censo determino que en el 2016 el parque automotor fue de 2.056.213 vehículos matriculados, debido a las facilidades de adquisición de nuevos vehículos, haciendo que los talleres mecánicos, lavadoras automotrices, servicio de cambio de aceite automotriz, concesionarios de vehículos y la industria en general, es donde se concentra el almacenamiento de los aceites residuales, la mayor concentración y así mismo las autoridades han planificado y controlado los desechos de aceites residuales en quito, Ibarra, cuenca, las demás ciudades están planificando estrategias para optimizar el manejo de este elemento contaminante. (Instituto Nacional de Estadística y Censos, 2018). Cabe destacar, que el crecimiento de la población de Riobamba, al igual que el parque automotor y por ende la comercialización de los aceites usados para los mantenimientos de estos vehículos está generando una problemática a la sociedad debido a la contaminación ambiental, la irresponsabilidad de la eliminación de desechos generados en estos vehículos y especialmente el no tener una normativa de reutilización de estos desechos, contribuyen a que se genere esta problemática. (Neira Altamirano, 2016)

A tal efecto, se investigaron las causas que limitan a los centros generadores de aceites que han cumplido con su vida útil, que practiquen de forma adecuada la disposición final de los mismos, y de esta forma aportar herramientas para la Gestión del manejo adecuado de aceites residuales, que sean útiles y aplicables en tiempo y espacio, contribuyendo a la capacitación de los involucrados con esta importante industria, para la concientización de la preservación de la salud humana y ambiental. Ante lo expuesto, este artículo se enfoca en el análisis del estado actual del manejo de los lubricantes residuales de los automotores, las aguas residuales que desecha desde los locales de mantenimiento de vehículos, así como también el espacio del suelo donde se realiza el cambio de aceite y principalmente el lugar en donde se recopilan los desechos de los lubricantes en estos centros dedicados al mantenimiento automotriz.

\section{Desarrollo}

En el trabajo realizado por Barrera y Romero (2015), sobre el "Diagnóstico de la contaminación ambiental causada por aceites usados provenientes del sector automotor y planteamiento de 
soluciones viables para el gobierno autónomo descentralizado del cantón azogues", manifiesta que el aceite lubricante usado automotriz categorizado como residuo líquido peligroso, es uno de los puntos a tratar por parte de todas la entidades e instituciones en las diferentes provincias y cantones en Ecuador, por tal motivo dichas entidades se han visto en la obligación de reducir el índice de contaminación causado por el aceite usado proveniente de los diferentes centros automotrices y establecimientos generadores de este desecho contaminante. En la que se determinó que una causa por la que se tiene un mal manejo de los desechos es debido a la falta de información proporcionada por el GAD municipal a los dueños y trabajadores de los talleres y lubricadoras, también sé determinó que se diseñe en los 66 centros automotrices no cuentan con un espacio suficiente para poder implementar zonas de almacenamiento seguro. También se determinó que la forma de transporte de estos residuos no es el adecuado, ya que no se cuenta con un camión cisterna que impediría la contaminación.

Así mismo, en la Universidad Central del Ecuador (2016), en la carrera de Ingeniería en Geología, Minas, Petróleos y Ambiental el proyecto titulado "Manejo ambiental adecuado de los desechos aceitosos en el Ecuador", se desarrolló un proyecto relacionado con las propuestas aplicable al manejo ambientalmente aceptable de los desechos aceitosos provenientes de la función lubricante, que resulta ser un producto disperso en el medio ambiente, manejado por la ciudadanía en los diferentes componentes sociales y productivos: talleres mecánicos, lavadoras de automotores, servicio de cambio de aceite automotriz, concesionarios de vehículos, transporte marítimo, actividad pesquera, la industria en general, incluyendo la hidrocarburífera como uno de los componentes de mayor impacto y aun en los hogares donde se hace cambios de aceite de los vehículos.

La investigación presentada por Coyago, Moromenacho, Martinez y Merchan (2017), en la que se hizo un análisis de los aceites a través de una prueba tribológica en un motor de gasolina con el aceite SAE 20W50, API SN, se conoció el desgaste de los componentes internos del motor, basándose en los límites condenatorios y sus rangos establecidos, observando que el primer aceite analizado generaba un mayor porcentaje de hollín que el segundo aceite analizado, afectando el desgaste prematuro de los componentes internos del motor. 
Por su parte, Delgado, Fygueroa y Sierra (2015), estudiaron el proceso de diseño y la construcción de un equipo de pruebas rápidas para el análisis de aceites usados de motores para evaluar el estado de degradación y contaminación de aceite usado mediante ensayos de crepitación, mancha de aceite, viscosidad y contenido de partículas, el cual consta de cuatro aparatos: placa de calentamiento, generador de manchas, viscosímetro de caída de bolas y detector de partículas ferrosas. El aporte fundamental del trabajo lo constituyen los dispositivos y métodos ideados tanto para medir con exactitud el tiempo de caída de la esfera del viscosímetro de bolas como para la detección de partículas ferromagnéticas. El equipo ha producido resultados satisfactorios comparados con los obtenidos en pruebas de laboratorio.

En el trabajo de investigación presentado por Prasetyowati (2016), en la que determino el valor de lubricantes de viscosidad cinemática que se ha utilizado a varias temperaturas y el uso a determinada distancia. Este estudio también pretende remediar mengtahui cómo el valor de la viscosidad cinemática del coche lubricante que se ha utilizado en una amplia gama de variación de la temperatura y el uso a determinada distancia. El líquido de viscosidad, en este caso los lubricantes, se puede determinar usando el viscosímetro de Redwood, para medir el tiempo de flujo requerido por $50 \mathrm{ml}$ de la muestra a una temperatura constante.

También, el trabajo de investigación presentado por Mohd Daud, Sharfi Najib, y Zahed,Mond (2016), indica que el aceite lubricante es uno de los elementos más importantes necesario para cada motor de automóvil con el fin de reducir la fricción entre las partes móviles en un motor de automóvil, reducir la temperatura del motor y evitar la entrada de contaminantes. Las personas tienen dificultades para identificar el nivel de degradación del aceite lubricante. Debido a eso, el aceite lubricante falso o reciclado aparece en el mercado. Se utilizó E-nose para superar la dificultad clasificando el perfil de olor patrón de la muestra de aceite lubricante. Los patrones se clasificaron utilizando la técnica de razonamiento basada en casos. El resultado de la clasificación muestra que CBR da un rendimiento de clasificación del 100\%, en la que se presentó el estudio de clasificación de aceites lubricantes a base de perfiles de olores utilizando el clasificador de Razonamiento basado en casos (CBR). 


\section{El Aceite Lubricante}

Beltrán (2012), expresa que los aceites lubricantes están constituidos por una base, la cual provee las características primarias de lubricación; la base puede ser mineral, sintéticas o vegetal, según la aplicación que se dé al aceite. Los lubricantes tienen como función primordial evitar el contacto directo entre superficies con movimiento relativo, reduciendo así la fricción y sus funestas consecuencias: calor excesivo, desgaste, ruido, golpes, vibración, etc. Básicamente en un lubricante, plantean Coyago y et al (2017), las propiedades dependen de su aceite base y ciertos tipos de aditivos empleados.

En cuanto a su gravedad y densidad relativa, anota Martínez (2011), que es una propiedad que usualmente se usa para identificar un cierto tipo de lubricante; es decir la densidad de un aceite a $15.60{ }^{\circ} \mathrm{C}$ y se calcula mediante la densidad del agua $0.99904 \mathrm{~g} / \mathrm{cm} 3$. Y el número de bases totales (TBN O BN). El TBN o BN es la cantidad de ácido clorhídrico $(\mathrm{HCl})$ en mg necesaria para neutralizar los componentes alcalinos de una muestra de $1 \mathrm{~g}$ de aceite. Se utiliza en aceites de motor (Martínez Pérez, 2011). Es decir, es el encargado de medir la cantidad de reserva antiácida del lubricante disponible para neutralizar los ácidos que forman la combustión, entendiendo la acidez total (TAN), como la cantidad de ácido expresado en términos del número equivalente de miligramos de hidróxido de potasio $(\mathrm{KOH})$ que es necesario para titular las bases fuertes presentes en un gramo de aceite, la cual se origina debido a la presencia de aditivos o compuestos ácidos que se forman durante ciertos procesos de oxidación y degradación. Entonces tiene como efecto la oxidación y la degradación con un aumento considerable a formar depósitos de barnices y lacas.

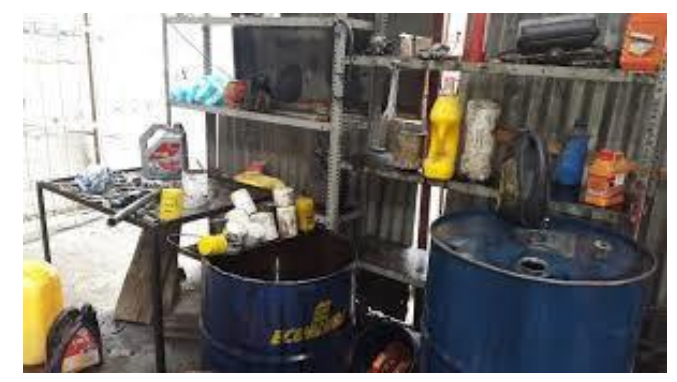

Figura 1. Almacenamiento inadecuado de aceite residual.

Al respecto, Barrera y Velecela (2015), indican que el punto de inflación de un aceite lo determina la temperatura mínima a la cual desprende vapores inflamables que se encienden en presencia de 
una llama o de punto incandescente. Este punto debe ser lo más alto posible para evitar que el aceite se incendie al estar en contacto con zonas del motor a altas temperaturas. Esta propiedad, según Suntaxi Beltrán (2012), tiene como función definir las propiedades de flamabilidad y la volatilidad a temperaturas elevadas.

Es de señalar que la Biodegradabilidad y toxicidad, según Martínez Pérez (2011), son dos propiedades del lubricante comparten características similares. La primera es una propiedad que influye demasiado en lo ambiental, ya que se involucra con los suministros de agua y el ambiente en general, y la toxicidad por otra parte es un problema y por tal motivo esta debe permanecer en un continuo seguimiento y atención.

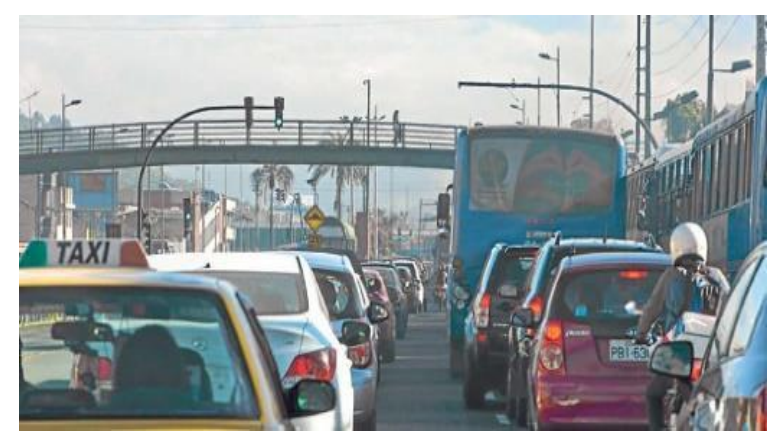

Figura 2. Incremento del parque automotor en Ecuador

Es de notar, que entre los daños ocasionados por los aceites usados de automotrices, está la contaminación, dado que este aceite contiene una variedad de sustancias ajenas al mismo, que causan su impureza y con ello una incorrecta lubricación para el cual fue fabricado. En este sentido, Suntaxi Beltrán (2012), expresa que una de las principales causas de que existían impurezas o contaminantes en el aceite, es debido a que en el momento de su fabricación hayan quedado pequeñas artes de partículas y residuos provenientes de las piezas o durante el mecanizado del mismo. O puede haber ocurrido también una contaminación tanto interna como externa dependiendo del entorno en el que se encuentre. 


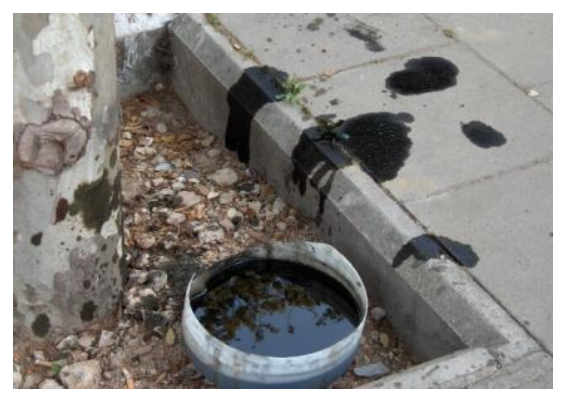

Figura 3. Contaminación de aceite automotriz

Mendoza Haro y Robles Salguero (2015), indican que el grado de contaminación, en el Ecuador por residuos peligrosos como es el aceite usado automotriz, es un tema de precaución para el país y para ciudades como lo es Riobamba. Debido a la gran demanda de vehículos en el país, se ha producido un aumento excesivo de los residuos de aceite lubricante. Además, agregando a esto la carencia de conocimiento de una gestión integral de las diferentes provincias del país.

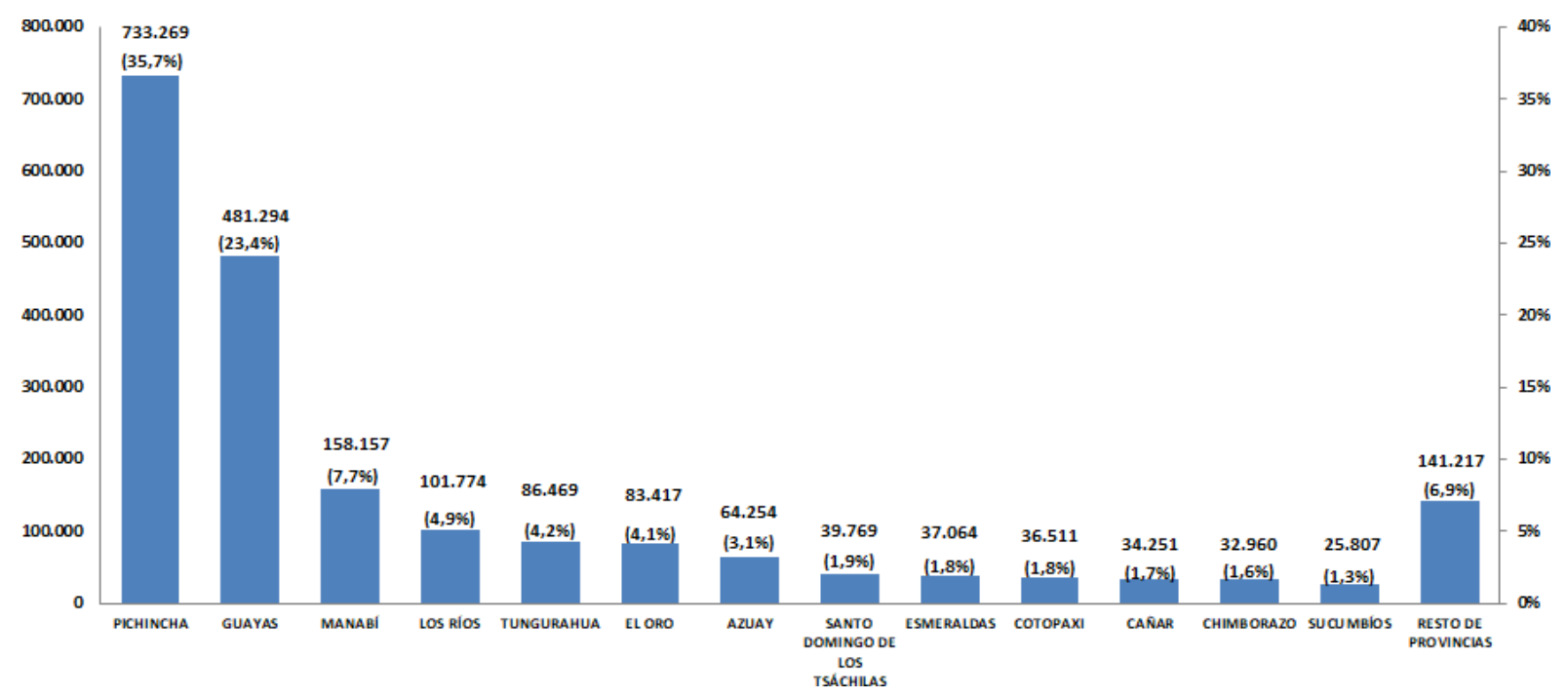

Figura 4. Vehículos matriculados en el año 2016

La falta de compromiso y dedicación, indican Suntaxi Beltrán (2012), por parte de talleres automotrices, lubricadoras, tecni-centros y demás localidades referentes a este medio en Ecuador; ha generado un incremento en el vertido incontrolado de aceite usado automotriz, en dichos centros 
ocasionando la muerte de plantas y causando malestar en la salud del ser humano y de las personas que residen cerca de estos establecimientos.

Esto se debe, según Torres Cobos (2014), a la inexistencia de infraestructuras que deberían ser parte de cada localidad o centros automotrices en todas las provincias y cantones del Ecuador. Cabe recalcar que ciertas ciudades del Ecuador como Loja, poseen una planta de re-refinamiento del aceite automotriz ubicada en el parque industrial de esta ciudad, la cual nunca logro funcionar a la perfección, ni tampoco logró obtener la licencia ambiental correspondiente y en la actualidad no funcionan. Los aceites lubricantes usados no tienen ningún control en la ciudad perjudicando así al ambiente y la salud. (Área Metropolitana Valle de Aburrá, 2006)

Según la Agencia de Protección Ambiental de los Estados Unidos (EPA, 2013), explica que un galón de aceite usado contamina a un millón de galones de agua, la misma que satisface las necesidades de cincuenta personas por un año.

\section{Grado de aceptabilidad de los aceites usados}

Los aceites lubricantes usados, para Coyago et al (2017), se pueden categorizar en niveles de aceptación tomando en cuenta aspectos como el grado de contaminación existente en dicho fluido. Y se pueden considerar como un recurso utilizable dependiendo de una clasificación que determine la necesidad de tratamiento que tenga el aceite; ajustándose a los niveles de contaminantes, entre estos:

- Si la cantidad de contaminantes existentes en el aceite lubricante usado es inferior o igual a los datos que presentan la tabla 1 , este puede ser reutilizado como combustible o como insumo industria, sin ninguna prohibición.

- Si existe presencia de contaminantes con cantidades mayores a los límites que nos da el manual, el aceite necesariamente debe ser transferido a un proceso para reducir el nivel de contaminantes existente en el lubricante.

- Si existe una gran concentración de policlorobifenilos o bifenilos policlorados (PCB’s) incluso mayor al límite establecido por el manual, debe someterse al aceite usado a un tratamiento especial que es la declorinación. 
Tabla 1. Concentración de sustancias máximas permisibles

\begin{tabular}{ll}
\hline SUSTANCIA & $\begin{array}{c}\text { CONCENTRACIÓN } \\
\text { MÁXIMA PERMISIBLE } \\
(\mathbf{m g} / \mathbf{K g}-\mathbf{p p m})\end{array}$ \\
\hline $\begin{array}{c}\text { Bifenilos } \\
\text { policlorados } \\
\text { (PCBs) }\end{array}$ & 50 \\
\hline $\begin{array}{c}\text { Halógenos } \\
\text { orgánicos } \\
\text { totales } \\
\text { (como cloro) }\end{array}$ & 1000 \\
\hline Arsénico & 5 \\
\hline Cadmio & 2 \\
\hline Cromo & 10 \\
\hline Plomo & 100 \\
\hline Azufre & $1.7 \%$ en peso \\
\hline
\end{tabular}

Una vez que los índices de contaminantes son estudiados, indican Coyago y etal (2017), se procede a dar paso para la utilización del aceite, dividiéndolo en 2 grupos: el primero es el aceite lubricante tratado, y el segundo es el aceite lubricante sin tratar.

El primer grupo se puede aprovechar en el campo industrial como combustible, asimismo una vez realizando diversos procesos de refinería al aceite se puede reutilizar, extendiendo una vez más su vida útil. El segundo grupo se puede utilizar en el campo energético solamente como combustible y en procesos productivos del cemento. Siendo así un aprovechamiento muy pobre en comparación con el primer grupo.

\section{Efectos causados por el aceite usado automotriz}

El aceite usado automotriz, expresan Daud, Najib, y Zahed, (2016) tiene un efecto peligroso y terminal para el ambiente y la salud del ser humano, debido a su consistencia puede provocar una serie de inconveniencias en los distintos medios donde es vertido o derramado, causando así una alteración total del entorno al cual se encuentra afectado. Este aceite usado, al ser expuesto completamente a la luz solar y al aire es capaz de emitir una gran cantidad de compuestos tóxicos, como lo son $\mathrm{Pb}, \mathrm{Zn}$ y $\mathrm{Cr}$. 
Los efectos que el aceite usado automotriz tiene, según Brow, Steve, Yana y Prior (2002), al entrar en contacto directo con los diferentes medios del ambiente son la tierra, en el aire y el agua, son los siguientes:

\section{- Efectos en la tierra}

El aceite al ser derramando en la tierra, provoca infertilidad en el suelo debido a que el aceite usado contiene hidrocarburos que producen la muerte del suelo y transforma la vegetación en inerte. Además, el aceite derramando en el suelo causa es efecto nocivo en el ambiente, ya que este al entrar en contacto con la luz del sol y ayuda del aire emana compuestos tóxicos, permitiendo de esta manera que estos compuestos sean filtrados a través del suelo y contaminen el mismo. (Widman International SRL, 2017)

Un problema común en los centros de cambio de aceites como lo son los tecni-centros, mecánicas, lubricadoras entre otras, no disponen de un plan de almacenamiento adecuado por lo que se ven en la necesidad de verter el aceite usando en coladeras, baldes y terrenos baldíos cercanos a sus establecimientos; causando así un problema en la salud de quienes habitan a sus alrededores. EL aceite usado derramado en el suelo tiene la capacidad de expandirse rápidamente, lo que ocasiona la formación de un film que no permite el ingreso de oxígeno lo que conlleva a lo que hoy en día se conoce como suelos infértiles o pocos productivos.

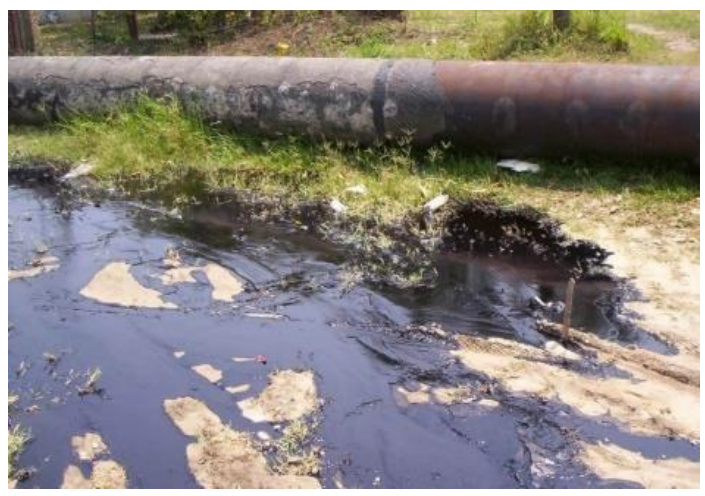

Figura 5. Contaminación de la tierra por aceite usado automotriz

\section{- Efectos en el aire}

Si el aceite usado se quema, solo o mezclado con el fuel-oíl, sin un tratamiento y un control adecuado origina importantes problemas de contaminación y emite gases muy tóxicos, debido a la 
presencia en este aceite de compuestos de plomo, cloro, fósforo, azufre, etc. Cinco litros de aceite quemados contaminan con plomo y otras sustancias nocivas $1.000 .000 \mathrm{~m} 3$ de aire, que es la cantidad de aire respirada por una persona durante tres años. (Suntaxi Beltrán, 2012)

\section{- Efectos en el agua}

Los diversos efectos que ocasiona el verter el aceite usado automotriz en el agua, ya sea de forma directa en ríos o por medio de vías de alcantarillado. En el caso de los talleres u servicios técnicos ubicados cerca de ellos, produce que el aceite se concentra demasiado en la superficie de estos ríos formando una capa impenetrable de oxígeno que causa la muerte de seres vivos que habitan en ese medio, así como animales terrestres que beben del lugar.

Por otro lado, al analizar el aceite usado automotriz se sabe que este contiene ciertas partículas que en combinación con el agua pueden fácilmente disolverse y filtrarse en las profundidades de los suelos acuáticos; con lo cual vienen a causar la muerte de la fauna y flora de ríos, mares u océanos al transcurso de los años.

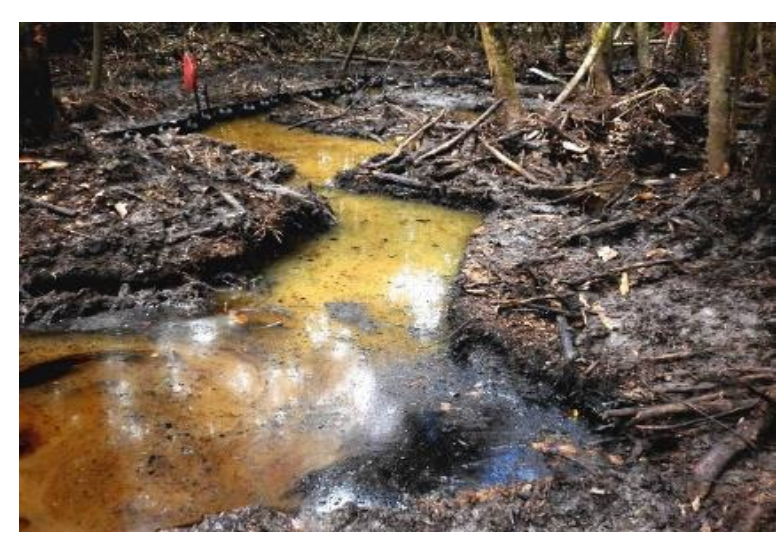

Figura 6. Aceite automotriz vertido en agua

\section{Metodología}

De acuerdo al objeto de estudio, el cual se relaciona con las aplicaciones de las teorías del análisis situacional del tratamiento de aceites automotrices residuales, esta se puede enmarcar dentro de una investigación de tipo descriptiva, ya que la misma se argumenta en lo planteado por Hurtado (2000), quien expresa que la investigación descriptiva permite exponer el evento estudiado, haciendo una enumeración detallada de sus características. Asimismo, estuvo basada en la revisión documental a través de la recopilación y análisis de investigaciones anteriores y de teorías que 
fundamentaron el estudio. La población sujeta a investigación, fueron las lubricadoras y talleres en la zona urbana del cantón Riobamba. Los resultados se obtuvieron a partir de visitas monitoreadas de seguimiento del destino de los aceites residuales en desuso por los automotrices.

\section{Conclusiones}

- El manejo inadecuado de los aceites que han sido reemplazados en los automóviles por las lubricadoras y mecánicas automotrices, se han dado por varios aspectos: falta de conocimiento de procedimientos técnicos para el manejo adecuado de este residuo y la composición química que estos poseen. Además, por la ausencia de un modelo de gestión de manejo de aceites usados y sus alternativas de reutilización.

- También se percibió la ausencia de vinculación entre la universidad, la industria y la sociedad, para crear compromisos con el manejo de dichos desechos que pueden ser reutilizados como combustible en la industria, en la que se propongan alternativas de manejo y tratamientos para su posible reutilización.

- Se constató que los centros dedicados al mantenimiento automotriz en la ciudad de Riobamba, no cuentan con datos históricos de planes de gestión para este fin, por tanto no se cuenta con indicadores de cantidad, calidad y propuestas de gestión por las autoridades.

\section{Referencias Bibliográficas}

Área Metropolitana Valle de Aburrá. (2006). Manual para el manejo Integral de Aceites Lubricantes. Medellín, Colombia. Obtenido de http://www.metropol.gov.co:9000/calidadaire/lsdocCombustibles/anexo_3_manual_alu_para_tall eres.pdf

Asamblea Nacional República del Ecuador. (2008). Constitución de la República del Ecuador. Montecirsti, Ecuador. Obtenido de http://www.asambleanacional.gob.ec/sites/default/files/documents/old/constitucion_de_bolsillo.p $\mathrm{df}$

Barrera Gallegos, L. A., y Velecela Romero, F. A. (2015). Diagnóstico de la contaminación ambiental causada por aceites usados provenientes del sector automotor y planteamiento de 
soluciones viables para el Gobierno Autónomo Descentralizado del Cantón Azogues. Tesis de pregrado.

Brow, J. Steve; Yana, Samuel F; Prior y Domanski A. (2002). Comparitive analysis of an automotive air conditioning systems operating with CO2 and R134a. International Journal of Refrigeration, 19-32.

Daud, S., Najib, M., y Zahed, N. (2016). Classification of lubricant oil odor-profile using casebased reasoning. Process and Control (ICSPC, 207-2012.

Delgado Sanabria, J. C., Fygueroa Salgado, S., y Sierra López, S. E. (2015). Diseño de un equipo de pruebas rápidas para aceites usados. INGE CUC.

EPCOS. (08 de 01 de 2017). eecs.oregonstate.edu. Obtenido de http://eecs.oregonstate.edu/education/docs/datasheets/10kThermistor.pdf

Grupo de Trabajo de Conservación de la Naturaleza, BWE. (2011). DOCUMENTO DE POSICION SOBRE CONSERVACIÓN DE LA NATURALEZA Y LA ENERGÍA EÓLICA DE FEDERAL. WINDENERGIE E.V.

Hurtado de Barrera, Jacqueline (2000). Metodologia de la Investigación Holística. Fundación Sypal. Caracas Venezuel.

Ingeniería en Geología, Minas, Petróleos y Ambiental - UCE. (06 de Mayo de 2016). Manejo ambiental adecuado delos desechos aceitosos en el Ecuador. Quito, Ecuador.

Instituto Nacional de Estadística y Censos. (01 de 04 de 2018). Anuario de estadística de transporte. Obtenido de http://www.ecuadorencifras.gob.ec/documentos/webinec/Estadisticas_Economicas/Estadistica\%20de\%20Transporte/2016/2016_AnuarioTransportes_ Resumen\%20Metodol\%C3\%B3gico.pdf

Martínez Pérez, F. (2011). Tribología Integral. México D.F: Limusa.

Mendoza Haro, E. I., y Robles Salguero, R. E. (2015). Reciclaje de aceites usados para transmisión de potencia en las industrias y talleres de servicio de la ciudad de Milagro, Ecuador. 160-165. 
Mohd Daud, S., Sharfi Najib, M., y Zahed, N. (2016). Classification of lubricant oil odor-profile using case-based reasoning.

Neira Altamirano, L. F. (2016). El impuesto verde y la reducción del parque automotor en la provincia de Chimborazo, durante el periodo 2016. Tesis de Pregrado. Riobamba: Universidad Nacional de Chimborazo.

Prasetyowati, R. (2016). determinacion del valor de lubricantes de viscosidad cinemática utilizado a varias temperaturas y el uso a determinada distancia.

Remache Coyago, A. P., Puente Moromenacho, E. G., Aulestia Martínez, R. D., y Noroña Merchán, M. V. (2017). Análisis tribológico en un motor de gasolina con dos marcas de lubricantes y la misma especificación. INNOVA Research Journal, 150 - 166.

Secretaría Nacional De Planificación Y Desarrollo. (2013). Plan Nacional para el Buen Vivir 20132017. Quito.

Suntaxi Beltrán, J. L. (2012). "Propuestas para el manejo de fluídos contaminantes de un taller automotriz en el sector sur del Distrito metropolitano de Quito". Tésis de Grado, Escuela Politécnica del Ejército, Latacunga, Ecuador. Obtenido de https://repositorio.espe.edu.ec/bitstream/21000/5088/1/T-ESPEL-0877.pdf

Torres Cobos, P. A. (2014). Diseño de un plan de recolección y el re-refinamiento de los aceites lubricantes usados en la ciudad de Loja. Tesis de pregrado

Widman International SRL. (5 de Mayo de 2017). Degradación. (Widman International SRL) Obtenido de Widman International SRL: http://www.widman.biz/Analisis/degradacion.htm 